\title{
Lidil
}

Revue de linguistique et de didactique des langues

\section{Acquisition des attaques branchantes (complexes) par des enfants bilingues simultanés portugais- français}

Acquisition of Branching Onsets by Simultaneous Portuguese-French Bilingual Children

Laetitia de Almeida, Yvan Rose et Maria João Freitas

\section{OpenEdition}

\section{Journals}

Édition électronique

URL : http://journals.openedition.org/lidil/3742

DOI : $10.4000 /$ lidil.3742

ISSN : $1960-6052$

Éditeur

UGA Éditions/Université Grenoble Alpes

Édition imprimée

Date de publication : 30 mai 2015

Pagination : 143-168

ISBN : 978-2-84310-297-4

ISSN : $1146-6480$

\section{Référence électronique}

Laetitia de Almeida, Yvan Rose et Maria João Freitas, « Acquisition des attaques branchantes (complexes) par des enfants bilingues simultanés portugais-français », Lidil [En ligne], 51 | 2015, mis en ligne le 30 novembre 2016, consulté le 20 avril 2019. URL : http://journals.openedition.org/ lidil/3742 ; DOI : 10.4000/lidil.3742 


\title{
Acquisition des attaques branchantes (complexes) par des enfants bilingues simultanés portugais-français
}

\author{
Laetitia de Almeida*, Yvan Rose** \\ et Maria João Freitas***
}

\begin{abstract}
Résumé
Dans cet article, nous présentons les problématiques théoriques de l'étude du développement simultané de deux langues et abordons les questions empiriques et méthodologiques associées. Nous analysons des données de 10 enfants bilingues portugais-français, âgés entre 3 et 4 ans, en nous concentrant sur leurs productions d'attaques branchantes (ou complexes) présentes dans des mots élicités à l'aide d'un protocole prédéfini. Nous comparons ces données à celles d'enfants monolingues et bilingues disponibles dans la littérature, en faisant référence aux résultats obtenus à travers différentes méthodologies (corpus longitudinaux $v s$ transversaux; données spontanées $v s$ induites). Nos résultats montrent que certains patrons sont communs à la plupart des bilingues évalués, notamment l'acquisition plus rapide des attaques branchantes en français par rapport au portugais. Nos résultats révèlent aussi la variation entre apprenants, notamment à propos de l'influence du type de consonne liquide (rhotique ou latérale). Nous montrons que cette variation ne peut être expliquée par un profil de dominance linguistique différent parmi les enfants concernés. Nos données suggèrent également que la question des influences inter-linguistiques ne peut être approchée facilement à l'aide de corpus de données recueillies transversalement, parce que seul un suivi longitudinal du développement peut réellement rendre compte de telles influences.
\end{abstract}

\section{ABSTRACT}

In this paper, we discuss theoretical issues involved in bilingual development, and address related empirical and methodological questions.

* INSERM U930 Imagerie et Cerveau, Université François-Rabelais de Tours.

** Department of Linguistics, Memorial University of Newfoundland.

*** Faculdade Letras, Centro de Linguística, Universidade de Lisboa. 
We analyze production data from 10 Portuguese-French bilingual children, between 3 and 4 years of age, focusing on their production of branching (complex) onset in words elicited through a pre-defined protocol. We compare these data to those from monolingual and bilingual learners available through the literature, in light of the methods used to obtain these data (longitudinal vs cross-sectional approaches to data gathering; elicited vs spontaneous data production). Our results suggest that certain production patterns are common to most bilingual learners, for example the relatively faster rate of acquisition of branching onsets in French than in Portuguese. Our results also reveal variation between learners, for example concerning the type of liquid (rhotic or lateral) present within the onset cluster. We argue that this variation cannot be explained through a measure of linguistic dominance. Further, our data suggest that cross-linguistic influences cannot easily be studied through cross-linguistic data, as only a longitudinal tracking of phonological development can truly be helpful to uncover the extent of these influences.

\section{Développement bilingue}

Le développement simultané de deux langues par les enfants grandissant en milieu bilingue pose une série de problèmes théoriques particuliers, notamment en ce qui concerne la présence d'éventuelles interactions entre ces deux langues (Müller \& Hulk, 2001; Paradis \& Genesee, 1996). Bien que distinctes à plusieurs égards, les deux langues cibles de l'apprenant partagent aussi un ensemble plus ou moins important de propriétés grammaticales, par exemple au niveau de leur inventaire de sons ou du type de constructions lexicales ou morphosyntaxiques qu'elles contiennent (De Houwer, 2009). Il convient donc de comparer le développement bilingue au développement monolingue de chacune des langues de l'apprenant.

Ceci permet entre autres d'identifier les patrons de développement phonologique qui dérivent des propriétés grammaticales de chaque langue et ceux qui résultent de l'interaction entre les deux langues, et qui donc ne devraient pas se retrouver chez les monolingues. Paradis et Genesee (1996) proposent que l'interaction linguistique peut assumer trois formats : le transfert d'une propriété linguistique spécifique à une langue dans l'autre; l'accélération, lorsqu'une structure est acquise plus rapidement par des apprenants bilingues que par des monolingues; ou au contraire, un retard de stabilisation d'une structure dans une des langues de l'enfant bilingue par rapport aux monolingues. Ces auteurs soulignent, que pour postuler l'existence d'une interaction linguistique 
en contexte de développement bilingue, celle-ci doit être systématique, c'est-à-dire se manifester de manière cohérente au cours d'une période étendue. Depuis, de nombreuses études ont mis en avant l'existence de telles interactions durant le développement linguistique bilingue. Traditionnellement, les études se penchant sur le sujet se sont basées sur un suivi longitudinal des productions spontanées d'un nombre réduit d'enfants (p. ex., Lleó, 2002 et travaux subséquents). Plus récemment, des études ont abordé ce sujet à partir de données transversales induites à partir d'un plus grand nombre d'enfants (p. ex. Fabiano-Smith $\&$ Barlow, 2009). Cependant, même dans ces derniers cas, le groupe d'enfants bilingues étudiés demeure relativement restreint (8 enfants dans certains cas) et il est dans tous les cas hétérogène : bilingues simultanés et successifs sont typiquement inclus dans un même groupe. En effet, il est virtuellement impossible de créer des groupes homogènes d'enfants bilingues, à cause du nombre élevé de facteurs extragrammaticaux pouvant potentiellement influencer le développement linguistique : d'éventuels effets découlant de la quantité d'exposition aux deux langues, la qualité de l'input que l'enfant reçoit, la préférence pour une des langues, la structure familiale et sociale supportant l'utilisation des langues, le prestige relatif des langues, ainsi que l'identification de l'enfant à chaque langue, entre autres facteurs (Grosjean, 2004). Un des défis pour la recherche sur le développement bilingue consiste donc à bien identifier ces facteurs, en comprendre les implications et tâcher d'en contrôler les effets dans la mesure du possible, dans des cadres expérimentaux.

C'est du point de vue de l'acquisition de la phonologie que nous approchons ces questions dans cet article, plus spécifiquement concernant l'acquisition d'attaques branchantes (constituées de deux consonnes) par des apprenants bilingues français-portugais (variantes européennes). Nous décrivons ces données, que nous comparons aussi à celles d'apprenants monolingues français et portugais documentés lors d'études antérieures. D'une part, comme nous le verrons, les systèmes phonologiques du français et du portugais sont assez similaires en ce qui concerne les propriétés des séquences pouvant constituer une attaque branchante. D'autre part, nous verrons aussi que les enfants monolingues français et portugais suivent des parcours de développement différents concernant ce constituant syllabique. Nous discuterons également des problèmes particuliers relevant d'influences inter-linguistiques durant le développement de ces deux langues en contexte de bilinguisme simultané. 
Plus généralement, nous explorerons cette question en comparant deux approches méthodologiques différentes (données longitudinales spontanées $v s$ transversales induites) et à la lumière de certains facteurs extra-grammaticaux inhérents au bilinguisme, notamment le type de bilinguisme (simultané) et la relation de dominance qui peut exister entre les deux langues d'un apprenant bilingue.

\section{Approche méthodologique}

Dans cette section, nous présentons notre corpus, ainsi que les méthodes et outils que nous avons adoptés pour le recueil, la transcription et l'analyse des données.

\subsection{Profil des enfants}

Nous avons constitué un corpus documentant les productions de dix enfants bilingues portugais-français. Ces enfants ont été recrutés au lycée français Charles Lepierre à Lisbonne en mai 2012 en fonction d'un critère général mais fondamental : celui d'avoir été exposé à la fois au français et au portugais à la maison depuis leur naissance. Les enfants analysés dans cet article constituent le groupe des enfants fréquentant la petite section de maternelle de l'établissement (âgés de 3 et 4 ans; cf. tableau 1). Deux autres groupes, chacun de dix enfants, ont été évalués en moyenne et grande sections. Pour cette étude, nous avons choisi de nous concentrer sur les enfants formant le groupe le plus jeune, pour pouvoir analyser leur développement phonologique précoce. Les parents qui ont accepté que leur enfant participe à cette étude ont rempli un questionnaire qui nous a permis de dresser un profil linguistique pour chacun des enfants. Le questionnaire parental distribué est la traduction française d'un questionnaire développé à l'Université de la Colombie-Britannique, dans le cadre du projet de recherche Cross-linguistic Child Phonology Project, sur le développement phonologique enfantin, à travers plusieurs langues ${ }^{1}$. Ce projet a pour objectif d'élaborer des outils d'évaluation de la phonologie pour un ensemble de langues, et s'articule autour d'une approche multilinéaire de la phonologie (p. ex. Bérubé, Bernhardt \& Stemberger, 2013).

1. Ce projet est coordonné par May Bernhardt et Joseph Stemberger et financé par le Conseil de recherches en sciences humaines du Canada (\#410-20090348). 
Le questionnaire développé dans le cadre de ce projet permet d'obtenir des informations générales sur l'enfant et son environnement familial (p. ex., son âge, le nombre de frères et sœurs). De plus, nous avons ajouté une section à ce questionnaire, spécifiquement pour la présente étude, afin d'obtenir des renseignements permettant de caractériser l'environnement linguistique de ces enfants bilingues. Ces renseignements concernent l'âge de l'enfant au moment de son premier contact avec les deux langues, la langue utilisée à la maison (par l'enfant et par les membres de sa famille), la quantité d'input reçue dans les deux langues quotidiennement (estimée en pourcentage), la quantité d'utilisation des deux langues par l'enfant, une éventuelle préférence de l'enfant pour une des langues, formulée en termes de sentiment d'être plus à l'aise dans une des langues.

Tous les enfants évalués sont des bilingues simultanés, exposés au portugais et au français depuis leur naissance. Neuf d'entre eux ont un parent locuteur natif de chacune de leur langue, qui s'adresse à l'enfant en utilisant sa langue maternelle. Une fois les informations issues du questionnaire réunies, nous avons analysé trois aspects en particulier : (i) $1(\mathrm{a} / \mathrm{es}$ ) langue(s) utilisée(s) à la maison (inclusivement avec les frères et sœurs), (ii) l'éventuelle préférence de l'enfant pour une des langues et (iii) la quantité d'exposition à chaque langue pour l'enfant. Ces trois critères nous ont permis de classer les enfants selon trois types de bilingues : équilibré (É), dominant en français (F), ou dominant en portugais (P). Ainsi, nous avons établi pour chaque enfant le profil correspondant au plus grand nombre de critères. Cette caractérisation est fournie pour chaque enfant dans le tableau 1 ci-après :

\begin{tabular}{|l|l|l|l|l|l|}
\hline Code & Âge & Langue du foyer & Préférence & Exposition & Dominance \\
\hline PF01 & $4 ; 3$ & + de français & français & + de français & français \\
\hline PF02 & $4 ; 3$ & + de portugais & portugais & moitié-moitié & portugais \\
\hline PF03 & $4 ; 3$ & + de portugais & aucune & moitié-moitié & équilibré \\
\hline PF04 & $3 ; 8$ & + de français & aucune & moitié-moitié & équilibré \\
\hline PF05 & $3 ; 9$ & les deux & aucune & moitié-moitié & équilibré \\
\hline PF06 & $3 ; 6$ & français & français & + de français & français \\
\hline PF07 & $3 ; 7$ & les deux & portugais & + de français & équilibré \\
\hline PF08 & $4 ; 0$ & les deux & aucune & + de français & équilibré \\
\hline PF09 & $4 ; 3$ & + de français & aucune & + de français & français \\
\hline PF10 & $3 ; 6$ & français & français & moitié-moitié & français \\
\hline
\end{tabular}

Tableau 1. - Description des participants (identifiants, âge et dominance linguistique). 
Les dix enfants évalués sont âgés de 3;6 à 4;3. Ils possèdent des profils de dominance linguistique variés : cinq enfants sont dominants en français, quatre enfants sont équilibrés et un seul présente une dominance en portugais. Il convient de noter que cette caractérisation a pour but d'établir des différences de profil linguistique en ce qui concerne la langue du foyer. En effet, ces enfants possèdent un profil généralement similaire : ils ont tous été exposés aux deux langues depuis leur naissance, grandissent au Portugal et sont scolarisés dans un établissement français. La majeure partie des enseignements dans cet établissement se déroule en français, bien que le personnel enseignant soit bilingue. Le personnel non enseignant est généralement lusophone. Ainsi, au sein de cet établissement, les enfants évoluent en milieu bilingue. Par contre, malgré la similitude du profil général de ces enfants, il faut tenir compte du fait qu'il est très difficile d'obtenir des groupes homogènes d'enfants bilingues, en partie à cause de l'importante variation interindividuelle que l'on observe dans tout type de population multilingue (De Houwer, 2009).

\subsection{Recueil de données}

Nous avons évalué ces dix enfants au cours de deux sessions séparées, une en français et l'autre en portugais. Les sessions se sont déroulées à l'école, soit au cours de la même journée (pour trois enfants : PF01, PF02 et PF03), soit sur deux jours consécutifs (pour six enfants : PF04, PF05, PF06, PF08, PF09 et PF10). Pour un enfant (PF07), des circonstances indépendantes de notre recherche ont imposé un intervalle d'une semaine entre les deux sessions.

Les sessions en portugais ont été conduites par une locutrice native de portugais ${ }^{2}$ et celles en français par une locutrice bilingue simultanée en français et portugais. Seuls l'enfant et l'expérimentateur étaient présents pendant les sessions.

Des chercheurs au Centre de linguistique de l'Université de Lisbonne ont adapté l'outil d'évaluation de la phonologie du Cross-linguistic Child Phonology Project pour son utilisation en français et en portugais européens. L'outil français a été adapté par le premier auteur (Almeida, 2013), à partir d'une mouture conçue pour l'évaluation de la

2. Nous tenons à remercier les deux locutrices en question : Margarida Ramalho et Ana Tanganho. 
phonologie du français du Manitoba (Bérubé, Bernhardt \& Stemberger, 2013). Il contient un total de 126 mots contrôlés à partir de plusieurs variables linguistiques : l'inventaire consonantique, la position dans le mot, le constituant syllabique, la position tonique, ainsi que la longueur du mot. Cette adaptation de l'outil original comporte 29 mots contenant des attaques branchantes; 11 de ces mots présentent la latérale [1] comme second membre de l'attaque branchante, alors que les 18 autres présentent la rhotique uvulaire [в] (cf. Bérubé, Bernhardt, Stemberger \& Almeida, 2014 pour plus de précisions sur les propriétés phonologiques des items retenus). L'outil portugais a été élaboré par Ramalho, Almeida et Freitas (2013). Il est contrôlé pour les mêmes variables linguistiques que l'outil français. Il contient 57 mots comportant des attaques branchantes : 11 de ces mots présentent la latérale [1] comme second membre d'une attaque branchante, alors que 46 mots présentent la rhotique $[r]$ dans cette position. Nous fournissons les listes de mots pour ces deux langues en annexe.

Sur le plan visuel, les outils d'évaluation pour chacune des langues prennent la forme de présentations en format PowerPoint, dans lesquelles sont présentées des images ${ }^{3}$ représentant les items lexicaux choisis pour leurs propriétés linguistiques ${ }^{4}$. L'ensemble de ces images forme une histoire que l'expérimentateur raconte à l'enfant. Avant de regarder les images, l'expérimentateur explique à l'enfant qu'il/elle doit l'aider à raconter l'histoire. Celle-ci est constituée entre autres de phrases porteuses de sens afin d'inciter l'enfant à nommer les items lexicaux choisis.

Un exemple d'image et de son protocole d'administration sont fournis, en guise d'illustration, en figure 1 ci-dessous.

3. L'éxécution graphique des deux outils a été financée par la Fondation pour la science et la technologie (FCT), dans le cadre du projet PEst-OE/LIN/ UI0214/2013.

4. Rappelons que seuls certains de ces items contiennent des attaques branchantes. 


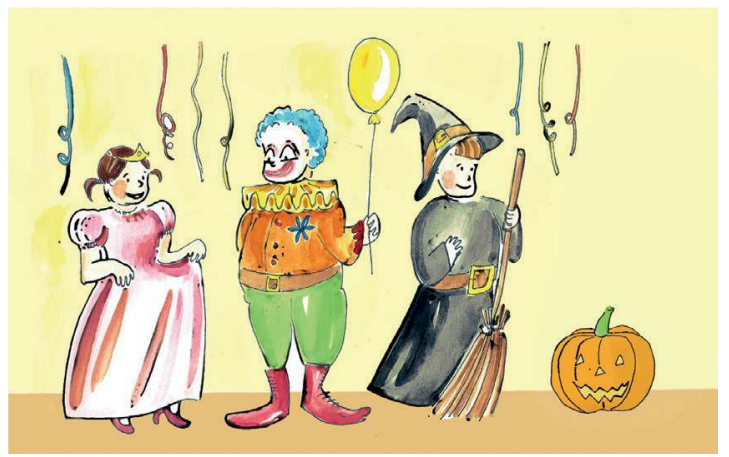

Figure 1. - Exemple d'une image et de ses instructions pour l'administration du protocole.

Instructions. - C'est carnaval et on va à un bal masqué. Marie s'est déguisée en (princesse) et porte une belle (robe). Guillaume s'est déguisé en (clown), Sandra en (sorcière). À côté d'elle il y a une (citrouille).

L'expérimentateur incite l'enfant à produire les items lexicaux cibles (représentés graphiquement entre parenthèses sur les instructions) à travers son intonation et en désignant l'image correspondante sur la diapositive. Si l'enfant n'arrive pas à produire l'item spontanément, l'expérimentateur lui fournit alors une aide phonétique en lui donnant la première partie du mot; si l'enfant n'y parvient toujours pas, l'expérimentateur prononce alors le mot et lui demande de le répéter. L'application de cet outil dure entre 10 et 15 minutes en français et entre 15 à 20 minutes en portugais, étant donné que l'outil portugais contient un plus grand nombre de mots cibles que celui du français. Malgré la durée de l'expérimentation, les enfants ont participé avec enthousiasme au protocole, sans signe de lassitude aucun : au contraire, plusieurs d'entre eux ont demandé à revoir l'histoire une fois celle-ci terminée. Les illustrations des deux outils ont été réalisées par une même dessinatrice. Cependant, les items lexicaux n'étant pas identiques dans les deux langues, les deux histoires sont distinctes. Les productions des enfants ont été enregistrées en format audio et transcrites ultérieurement. Nous décrivons ces étapes dans la prochaine section.

\subsection{Préparation et analyse des données}

Les données recueillies en français et en portugais pour les dix enfants ont été transcrites par le premier auteur à l'aide du logiciel Phon (<https://www.phon.ca>; Rose et coll., 2006; Rose \& MacWhinney, 
2014). Toutes les transcriptions ont été revues par un second linguiste : celles en français par le deuxième auteur et/ou par une locutrice native de français non impliquée dans cette étude, celles en portugais par le troisième auteur. Dans les rares cas où les différents transcripteurs ont obtenu des transcriptions divergentes, les deux transcripteurs concernés ont travaillé de concert de manière à obtenir une transcription consensuelle finale pour l'analyse. Dans la grande majorité des cas, les divergences concernaient des détails non pertinents pour la présente étude, notamment en portugais la transcription du degré d'antériorité des fricatives coronales. De plus, nous avons codé les données de manière à identifier les endroits où les enfants ont bénéficié d'indices supplémentaires pour produire la forme induite, de manière à vérifier que les patrons de production des enfants ne soient pas le résultat d'une imitation mais bien le reflet de leurs capacités phonologiques.

Comme nous n'avons observé aucune différence entre ces données et celles produites spontanément, nous combinons tous les types de production dans les résultats présentés ci-dessous.

Une fois les formes transcrites, nous les avons annotées à l'aide de fonctions spécialisées permettant l'identification des positions syllabiques de chaque segment ainsi que l'alignement, son par son, entre les formes cibles (adultes) et celles produites par les enfants, tel qu'illustré en figure 2 .

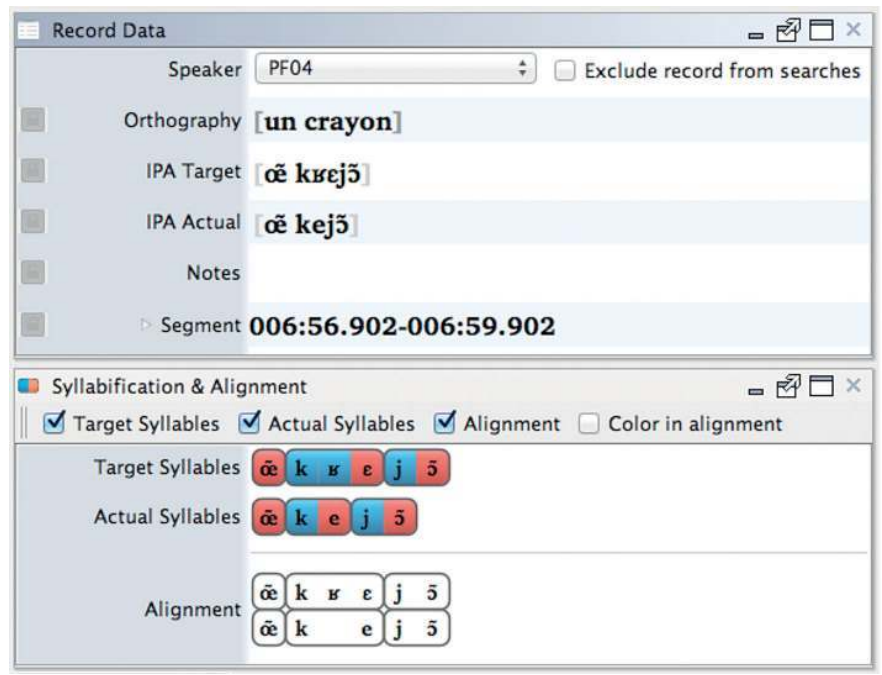

Figure 2. - Transcriptions et annotations dans Phon. 
Nous avons ensuite extrait les données pertinentes pour chacun des contextes discutés en détail ci-dessous.

\section{Propriétés des attaques branchantes}

D'un point de vue phonologique, le français et le portugais présentent des inventaires d'attaques branchantes relativement similaires. Entre autres détails, ces deux langues permettent des combinaisons d'obstruantes suivies par une liquide (latérale ou rhotique). Cependant, on retrouve des différences au niveau phonétique, surtout concernant la rhotique : celle-ci prend la forme d'une fricative uvulaire en français $(/ \mathrm{s} /)$ et d'un tap apical en portugais $(/ \mathrm{r} /)$, tel qu'illustré dans le tableau 2.

\begin{tabular}{|l|l|l|}
\hline & Portugais & Français \\
\hline Occlusive + rhotique & {$[\mathrm{p}]$ incesa } & {$[\mathrm{pr}]$ incesse } \\
\hline Occlusive + latérale & {$[\mathrm{pl}]$ ano } & {$[\mathrm{pl}]$ at } \\
\hline Fricative + rhotique & {$[\mathrm{fr}]$ anco } & {$[\mathrm{fb}] \mathrm{anc}$} \\
\hline Fricative + latérale & {$[\mathrm{fl}]$ or } & {$[\mathrm{fl}] \mathrm{eur}$} \\
\hline
\end{tabular}

Tableau 2. - Attaques branchantes en français et en portugais.

Dans les deux langues, une occlusive en tête d'attaque branchante peut être labiale, coronale ou vélaire. Cependant, si la seconde consonne est latérale, celle-ci ne peut être précédée d'une coronale. Aussi, une fricative en tête d'attaque ne peut être que labiale. Finalement, toutes les attaques branchantes sont permises en initiale et en milieu de mot, excepté [vr] en portugais, lequel n'est attesté qu'en milieu de mot (voir, p. ex., Goad \& Rose, 2004; Mateus \& Andrade, 2000; Rice, 1992 au sujet des implications théoriques de ces restrictions distributionnelles).

\section{Acquisition des attaques branchantes}

Dans cette section, nous abordons la question de l'acquisition des attaques branchantes en français et en portugais. Nous débutons avec un survol des études antérieures portant sur l'acquisition de ces attaques par des apprenants monolingues. Nous enchainons avec une étude longitudinale récente portant sur l'acquisition de ces mêmes attaques par une apprenante bilingue français-portugais. 


\subsection{Apprenants monolingues}

$\mathrm{Si}$ on considère les données d'apprenants monolingues, les attaques branchantes sont en effet acquises de manière assez différente selon ces deux langues. Au stade initial, les apprenants du français et du portugais se ressemblent en ce qu'ils réduisent généralement les attaques branchantes cibles en attaques simples, préservant la consonne obstruante, tel qu'exemplifié en (1) (Freitas, 1997, 2003; Rose, 2000), un comportement noté aussi chez les apprenants d'autres langues comme l'anglais et le néerlandais (Barlow, 1997; Fikkert, 1994; Smith, 1973; Spencer, 1986).

(1) Réduction d'attaques branchantes:

a. Français (Rose, 2000)

\begin{tabular}{|c|c|c|c|}
\hline pleure & |pl|eure & [pœ:] & (Clara, $1 ; 7.27)$ \\
\hline clown & |kl|own & [kon] & (Théo, 2;05.11) \\
\hline brisé & |bs|isé & [bœ: 'çi:] & $\begin{array}{l}\text { (Clara, 1;7.27) } \\
\text { (Théo } 2 \cdot 4 \text { ) }\end{array}$ \\
\hline
\end{tabular}

b. Portugais (Freitas, 2003)

$\begin{array}{lllll}\text { creme } & |\mathrm{kr}| \mathrm{eme} & {[\text { 'ke] }} & \text { (Inês, 1;5.11) } & \text { 'crème' } \\ \text { letras } & \text { le|tr|as } & {\left[\text { 'cte } \int\right]} & \text { (Marta, 1;11.10) } & \text { 'lettres' } \\ \text { quatro } & \text { qua|tr|o } & {[\text { 'atu] }} & \text { (João, 2;1.23) } & \text { 'quatre' }\end{array}$

C'est aux stades ultérieurs que les apprenants des deux langues se démarquent. En comparaison avec les apprenants du français, ceux du portugais acquièrent leurs attaques branchantes plus tardivement et aussi passent souvent par un stade d'épenthèse vocalique entre les deux consonnes de la séquence cible (Freitas, 1997, 2003). Freitas, qui a suivi et documenté longitudinalement sept enfants âgés de $0 ; 10$ à $3 ; 7$ ans, remarque que les attaques branchantes peuvent émerger tardivement, n'étant pas toutes acquises même chez les enfants les plus âgés. De plus, elle remarque également que les enfants produisent un nombre élevé d'épenthèses vocaliques entre les deux membres de l'attaque. Par exemple, Luis, à 2;4, produit $67 \%$ d'épenthèses et $33 \%$ de formes cibles (Freitas, 2003, p. 37), comme en témoignent les exemples en (2).

(2) Épenthèse vocalique en portugais (Freitas, 2003):

$\begin{array}{lllll}\text { zebra } & \text { ze }|\mathrm{br}| \mathrm{a} & \text { ['sepire] } & \text { (Luís, 2;5.27) } & \text { 'zèbre' } \\ \text { bicicletas } & \text { bici|kl|etas } & \text { [bisiki'lete }] & \text { (Laura, 3;1.6) } & \text { 'vélo' } \\ \text { cobra } & \text { co|br|a } & \text { ['kobire] } & \text { (Pedro, 3;5.18) } & \text { 'serpent' }\end{array}$


À notre connaissance, ce stade n'a jamais été observé systématiquement chez les apprenants de français, malgré quelques attestations sporadiques d'épenthèse notées dans des études longitudinales et transversales (Kehoe, Hilaire-Debove, Demuth \& Lleó, 2008; Rose, 2000 ; dos Santos, 2007). Nous notons aussi que les attaques branchantes comportant des latérales tendent à être acquises par les apprenants du français avant celles comportant une rhotique (Kehoe, Hilaire-Debove, Demuth \& Lleó, 2008; dos Santos, 2007). Les résultats des monolingues portugais sont variables : alors que des données transversales de 723 enfants montrent une stabilisation plus précoce des attaques branchantes de type $\mathrm{Cl}$ (Mendes, Afonso Lousada \& Andrade, 2009), des données longitudinales de sept enfants suggèrent que la qualité de la liquide n'influence pas la vitesse d'acquisition de l'attaque (Almeida \& Freitas, 2010). Les études longitudinales et transversales menées sur des enfants monolingues portugais s'accordent pour dire que les attaques branchantes ne sont pas totalement acquises en portugais avant l'âge de cinq ans (Mendes, Afonso, Lousada \& Andrade, 2009).

\subsection{Bilinguisme français-portugais}

Une étude de cas a été menée sur une enfant bilingue simultanée portugais-français, grandissant au Portugal, exposée au français et au portugais à travers ses parents, chacun locuteur natif d'une de ces deux langues (Almeida, 2011). Cette enfant, dont les productions sont documentées longitudinalement entre les âges de $1 ; 0$ à $3 ; 10$, montre des comportements linguistiques équilibrés dans ses deux langues. Cependant, elle n'acquiert pas les attaques branchantes contenues dans les mots de ces deux langues de manière autonome. Ces groupes consonantiques sont acquis de manière précoce et au cours de la même période dans les deux langues. Ainsi, bien que l'âge de stabilisation ne coïncide pas forcément dans les deux langues (les attaques branchantes ayant tendance à devenir stables plus tôt en français qu'en portugais), les patrons de développement sont identiques. Dans chacune de ses deux langues, l'enfant acquiert les séquences $\mathrm{Cl}$ avant les séquences $\mathrm{Cr}$, comme illustré dans les exemples en (3) ci-dessous.

(3) Production d'attaques $\mathrm{Cl}$ et réduction d'attaques $\mathrm{Cr}$ (Almeida, 2011):

a. Français

plein $\quad \mid$ pl $\mid \quad[p l \tilde{\varepsilon}] \quad($ Barbara, 2;9)

très $\quad \mid$ tв $\varepsilon \mid \quad[\mathrm{t} \varepsilon] \quad($ Barbara, 2;9) 


\section{b. Portugais}

Pluto |'plutu| ['pluu] (Barbara, 2;7) 'Pluto' (pers. de dessin animé) grande |'gredi| ['gẽedi] (Barbara, 2;8) 'grand'

De plus, aucun patron systématique d'épenthèse vocalique n'est observé, ni en français ni en portugais. En somme, les patrons de développement des attaques branchantes observés chez cette enfant sont généralement similaires à ceux des monolingues français. Ce parallélisme développemental suggère une interaction interlinguistique, laquelle se traduit par une accélération du développement des attaques branchantes en portugais, que l'enfant maitrise vers l'âge de trois ans, de manière beaucoup plus précoce que les apprenants monolingues portugais, qui ne maitrisent généralement pas ces attaques avant l'âge de cinq ans (Mendes, Afonso, Lousada \& Andrade, 2009).

Cependant, ces résultats découlent de l'observation longitudinale de données spontanées d'une seule enfant. Il est donc pertinent de vérifier si ce patron de développement peut être généralisable à d'autres enfants bilingues. Les résultats ci-dessous constituent un premier pas dans cette direction.

\subsection{Acquisition des attaques branchantes par des bilingues français-portugais}

Dans cette section, nous présentons les résultats de la production de mots contenant des attaques branchantes en français et en portugais, à partir d'une tâche d'élicitation de mots, à l'aide des outils décrits en section 2.2, auprès de dix enfants bilingues français-portugais. Nous présentons les résultats obtenus pour chaque enfant individuellement, de manière à pouvoir observer une éventuelle variation entre ces participants. Les données obtenues pour le portugais et pour le français sont exposées séparément, de même que les résultats pour les productions d'attaques cibles de type $\mathrm{Cl}$ et $\mathrm{Cr}$ dans chacune de ces langues.

Dans chaque tableau présenté ci-après, nous illustrons la proportion relative des productions cibles des attaques branchantes, des cas d'élision de la liquide et des cas d'épenthèse vocalique. Nous considérons qu'une attaque branchante est produite conformément à la cible lorsque les deux membres de l'attaque sont produits correctement. Nous considérons qu'une attaque branchante est acquise lorsque ses taux de productions correctes atteignent $80 \%$. Exception faite de la substitution de voisement de la fricative uvulaire en français, toute substitution de l'un des deux membres de l'attaque (y compris des substitutions de 
voisement ou de lieu d'articulation de la première consonne) est considérée comme incorrecte et regroupée sous l'étiquette «autres» dans l'exposé des résultats. Nous avons aussi regroupé d'autres productions possibles sous le libellé «autres». Il peut s'agir de l'élision de la première consonne, ou d'autres productions marginales dans le corpus étudié. Nous avons tenu compte de la totalité des attaques branchantes produites par chaque enfant qui ne correspond pas toujours au nombre attendu pour chaque séquence : certains enfants n'ont pas produit de mot cible, même après incitation à sa répétition; parallèlement, certains enfants ont, à quelques reprises, répété un ou plusieurs items.

Dans un premier temps, nous présentons dans le tableau 3 les résultats de la production des attaques branchantes du type $\mathrm{Cl}$ en français pour les dix enfants évalués. Rappelons qu'il existe 11 mots contenant cette structure dans l'outil français.

\begin{tabular}{|c|c|c|c|c|c|c|c|c|c|c|c|}
\hline & PF01 & PF02 & PF03 & PF04 & PF05 & PF06 & PF07 & PF08 & PF09 & PF10 & Total \\
\hline Total & 11 & 12 & 10 & 11 & 11 & 9 & 10 & 11 & 10 & 10 & 105 \\
\hline Cible & 11 & 10 & 10 & 9 & 1 & 7 & 8 & 9 & 8 & 10 & 83 \\
& $(100 \%)$ & $(83 \%)$ & $(100 \%)$ & $(82 \%)$ & $(9 \%)$ & $(78 \%)$ & $(80 \%)$ & $(82 \%)$ & $(80 \%)$ & $(100 \%)$ & \\
\hline Élision C2 & 0 & 1 & 0 & 1 & 10 & 1 & 1 & 1 & 1 & 0 & 16 \\
\hline Épenthèse & 0 & 0 & 0 & 0 & 0 & 0 & 0 & 1 & 0 & 0 & 1 \\
\hline Autre & 0 & 1 & 0 & 1 & 0 & 1 & 1 & 0 & 1 & 0 & 5 \\
\hline
\end{tabular}

Tableau 3. - Attaques $\mathrm{Cl}$ en français.

Neuf enfants sur dix produisent correctement les attaques branchantes $\mathrm{Cl}$ en français dans approximativement $80 \%$ ou plus des cas observés. En outre, une certaine variation entre les enfants est observable : trois enfants produisent systématiquement ces attaques branchantes de manière correcte (PF01, PF03 et P10); six enfants produisent correctement ces attaques branchantes dans la grande majorité des cas (PF02, PF04, PF06, PF07, PF08, PF09); et un enfant ne produit pratiquement jamais ce type d'attaques branchantes (une seule production correcte; PF05). Notons aussi que, parmi les trois enfants qui produisent systématiquement les séquences $\mathrm{Cl}$ conformément à la cible, on trouve un des enfants les plus jeunes de notre groupe (PF10; 3;6).

Comme nous l'illustrons en (4), dans les cas où les séquences $\mathrm{Cl}$ ne sont pas correctement produites, les enfants ont tendance à préserver la première consonne et à élider la latérale (systématiquement dans le cas de PF05), ou bien ils ont recours à d'autres types de productions, 
comme au contraire à préserver la latérale. Finalement, nous observons un unique cas d'épenthèse vocalique (produit par PF08).

(4) Exemples de production d'attaques branchantes de type $\mathrm{Cl}$ en français :

$\begin{array}{lllll}\text { Production cible } & \text { bleu } & \mid \text { blø } \mid & {[\text { blo] }} & \text { PF02, 4;3 } \\ \text { Élision latérale } & \text { plage } & \mid \text { plaz } \mid & \text { [paz] } & \text { PF05, 3;9 } \\ \text { Élision obstruante } & \text { glace } & \mid \text { glas } \mid & {[\text { las }]} & \text { PF09, 4;3 } \\ \text { Épenthèse vocalique } & \text { blanc } & \mid \text { blã } \mid & {[\text { bălã] }} & \text { PF08, 4;0 }\end{array}$

Nous passons à présent aux résultats de la production des 18 mots contenant des attaques branchantes du type $\mathrm{Cr}$ en français, rapportés dans le tableau 4 ci-dessous.

\begin{tabular}{|c|c|c|c|c|c|c|c|c|c|c|c|}
\hline & PF01 & PF02 & PF03 & PF04 & PF05 & PF06 & PF07 & PF08 & PF09 & PF10 & Total \\
\hline Total & 16 & 19 & 18 & 18 & 15 & 19 & 19 & 20 & 16 & 19 & 179 \\
\hline Cible & $\begin{array}{c}15 \\
(94 \%)\end{array}$ & $\begin{array}{c}9 \\
(47 \%)\end{array}$ & $\begin{array}{c}13 \\
(72 \%)\end{array}$ & $\begin{array}{c}14 \\
(78 \%)\end{array}$ & $\begin{array}{c}1 \\
(7 \%)\end{array}$ & $\begin{array}{c}16 \\
(84 \%)\end{array}$ & $\begin{array}{c}17 \\
(89 \%)\end{array}$ & $\begin{array}{c}15 \\
(75 \%)\end{array}$ & $\begin{array}{c}12 \\
(75 \%)\end{array}$ & $\begin{array}{c}13 \\
(68 \%)\end{array}$ & 125 \\
\hline Élision C2 & 0 & 4 & 0 & 2 & 12 & 1 & 0 & 1 & 1 & 0 & 21 \\
\hline Autres & 1 & 6 & 5 & 2 & 2 & 2 & 2 & 4 & 3 & 6 & 33 \\
\hline
\end{tabular}

Tableau 4. - Attaques Cr en français.

Nous observons une plus grande variation entre enfants dans la production des attaques $\mathrm{Cr}$, incluant le fait qu'aucun enfant n'arrive à les produire systématiquement, contrairement à ce qui a été observé pour les attaques $\mathrm{Cl}$. Les dix enfants évalués présentent trois comportements possibles : trois enfants ont un taux de production cible des structures Cr supérieur à 80\% (PF01, PF06 et PF07); six enfants présentent entre $47 \%$ et $80 \%$ de productions correctes (PF02, PF03, PF04, PF08, PF09, PF10); enfin, un enfant présente moins de $10 \%$ de productions correctes de cette structure (PF05). Encore une fois, parmi les trois enfants qui présentent le nombre le plus élevé de production cible se trouvent deux des enfants les plus jeunes : PF06 et PF07, âgés de 3;6 et 3;7, respectivement.

Lorsque les attaques branchantes du type $\mathrm{Cr}$ ne sont pas correctement produites en français, les enfants ont tendance à élider la liquide et à conserver la première consonne, à substituer une des deux consonnes de l'attaque cible (p. ex., son voisement, mode, ou lieu d'articulation), ou à élider la première consonne et à préserver la liquide. Par contre, aucune épenthèse vocalique n'est attestée entre les deux membres de l'attaque dans notre corpus. Ces différents patrons de production sont exemplifiés en (5). 
(5) Exemples de production d'attaques branchantes de type $\mathrm{Cr}$ en français :

\begin{tabular}{|c|c|c|c|c|}
\hline ction cible & trois & |tьwa| & [tьwa] & \\
\hline & froid & & [fwa] & PГUL, 4 \\
\hline & & |gкәnиј| & 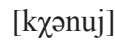 & $\mathrm{PF}$ \\
\hline lision obstru & bruit & |вкці| & [вwi] & $\mathrm{FC}$ \\
\hline
\end{tabular}

En résumé, nos données concernant la production d'attaques branchantes en français mettent en relief plusieurs faits. Premièrement, les attaques branchantes du type $\mathrm{Cl}$ sont plus souvent produites correctement que celles du type $\mathrm{Cr}$. Deuxièmement, l'épenthèse vocalique entre les deux membres de l'attaque n'est virtuellement pas attestée (un seul cas répertorié, pour une suite $\mathrm{Cl}$ cible). Finalement, on observe une variation considérable entre les enfants : six d'entre eux ont acquis les deux types d'attaque branchante (PF01, PF04, PF06, PF07, PF08, PF09); trois autres enfants présentent un taux supérieur de production correcte pour les attaques de type $\mathrm{Cl}$ par rapport à celles du type $\mathrm{Cr}$ (PF02, PF03 et PF10), alors qu'un enfant ne produit pratiquement aucune attaque branchante conformément à la cible (PF05).

Concernant la production d'attaques branchantes en portugais, nous commençons par les séquences du type $\mathrm{Cl}$, exposées dans le tableau 5 . Rappelons que l'outil portugais contient 11 mots comportant ce type d'attaque.

\begin{tabular}{|c|c|c|c|c|c|c|c|c|c|c|c|}
\hline & PF01 & PF02 & PF03 & PF04 & PF05 & PF06 & PF07 & PF08 & PF09 & PF10 & Total \\
\hline Total & 12 & 11 & 13 & 8 & 6 & 10 & 12 & 9 & 7 & 9 & 97 \\
\hline Cible & 5 & 5 & 12 & 5 & 0 & 4 & 7 & 3 & 3 & 4 & 48 \\
& $(42 \%)$ & $(45 \%)$ & $(92 \%)$ & $(63 \%)$ & $(0 \%)$ & $(40 \%)$ & $(58 \%)$ & $(33 \%)$ & $(43 \%)$ & $(44 \%)$ & \\
\hline Élision C2 & 4 & 1 & 0 & 0 & 4 & 5 & 3 & 4 & 1 & 3 & 25 \\
\hline Épenthèse & 3 & 3 & 1 & 1 & 0 & 0 & 0 & 1 & 3 & 0 & 12 \\
\hline Autre & 0 & 2 & 0 & 2 & 2 & 1 & 2 & 1 & 0 & 2 & 12 \\
\hline
\end{tabular}

Tableau 5. - Attaques $\mathrm{Cl}$ en portugais.

Tout d'abord, nous notons qu'aucun enfant ne présente une production correcte systématique des attaques branchantes du type $\mathrm{Cl}$ en portugais, contrairement à ce qui a été observé en français. Tout comme précédemment, il existe une certaine variabilité de comportements observables parmi les enfants évalués : un seul enfant (PF03) produit plus de $80 \%$ de formes cibles $\mathrm{Cl}$; huit enfants présentent des taux de productions correctes variables, entre $33 \%$ et $63 \%$. Enfin, un enfant n'est arrivé à produire aucune attaque $\mathrm{Cl}$ en portugais (PF05). 
Lorsqu'ils ne parviennent pas à produire correctement des séquences $\mathrm{Cl}$, la majorité des enfants préserve la première consonne de l'attaque au détriment de la liquide. Cependant, et contrairement à ce qui a été observé pour le français, l'épenthèse vocalique, bien qu'attestée peu fréquemment, est attestée chez six des dix enfants évalués (12 cas sur les 97 productions répertoriées). Dans ces cas, la voyelle épenthétique est généralement de type 'schwa' (i.e. centrale et non arrondie, p. ex. [i] ou $[\mathfrak{e}]$ ), similaire à ce qu'on retrouve dans les cas d'épenthèse chez les apprenants monolingues du portugais.

(6) Exemples de production d'attaques branchantes de type $\mathrm{Cl}$ en portugais :

Production cible flauta |'flawte| ['flute] PF04,3;8 'flûte'

Élision latérale flor |'flor| ['fo:] PF05, 3;9 'fleur'

Épenthèse clube |'klubi| ['kilub] PF04, 3;8 'club'

Substitution latérale ciclista |si'klifte| [și'kwijtte] PF07,3;7 'cycliste'

Les résultats concernant la production de mots contenant des attaques branchantes du type $\mathrm{Cr}$ sont exposés dans le tableau 6. L'outil portugais contient 46 mots possédant cette structure.

\begin{tabular}{|c|c|c|c|c|c|c|c|c|c|c|c|}
\hline & PF01 & PF02 & PF03 & PF04 & PF05 & PF06 & PF07 & PF08 & PF09 & PF10 & Total \\
\hline Total & 45 & 46 & 46 & 31 & 29 & 40 & 38 & 42 & 39 & 45 & 401 \\
\hline Cible & 0 & 1 & 35 & 10 & 0 & 13 & 26 & 19 & 18 & 18 & 140 \\
& $(0 \%)$ & $(2 \%)$ & $(76 \%)$ & $(32 \%)$ & $(0 \%)$ & $(33 \%)$ & $(68 \%)$ & $(45 \%)$ & $(46 \%)$ & $(40 \%)$ & \\
\hline Élision C2 & 8 & 40 & 7 & 10 & 24 & 13 & 6 & 10 & 6 & 11 & 135 \\
\hline Épenthèse & 0 & 0 & 1 & 2 & 0 & 11 & 2 & 5 & 7 & 6 & 34 \\
\hline Autre & 37 & 5 & 3 & 9 & 5 & 3 & 4 & 8 & 8 & 10 & 92 \\
\hline
\end{tabular}

Tableau 6. - Attaques Cr en portugais.

Une première observation est qu'aucun des dix enfants évalués ne présente plus de $80 \%$ de productions correctes des attaques branchantes $\mathrm{Cr}$ en portugais. En réalité, sept des enfants présentent des taux variables de production correcte, entre $32 \%$ et $76 \%$, alors que trois enfants n'arrivent pas à produire cette structure (PF01, $\mathrm{PF} 05$ et $\mathrm{PF} 02$, qui en a produit une seule à partir de 46 mots cibles).

L'élision de la liquide lorsque l'attaque n'est pas bien produite se retrouve dans les productions de tous les enfants observés. Tout comme pour les séquences $\mathrm{Cl}$, l'épenthèse vocalique, bien que peu fréquente, est attestée chez sept des dix enfants évalués (34 cas sur les 401 productions répertoriées). Tout comme pour $\mathrm{Cl}$, la voyelle épenthétique est généralement de type schwa. Notons qu'il existe de nombreux cas 
de substitution de la vibrante / $/$ / par la rhotique uvulaire [в]. Dans le cas de PF01, cette substitution est systématique : soit cette enfant élide la vibrante, soit elle la remplace par [в] ou par sa contrepartie non voisée $[\chi]$.

(7) Exemples de production d'attaques branchantes de type $\mathrm{Cr}$ en portugais :

\begin{tabular}{|c|c|c|c|c|}
\hline Production cible & cruz & & ['kruf] & PF09, 4;3 'croix' \\
\hline & 'pedre & |'pedre| & ['pede] & PF10, 3;6 'pierre' \\
\hline & livros & |'livruf| & ['liviruf] & PF08, 4;0 'livres' \\
\hline
\end{tabular}

Les données concernant la production d'attaques branchantes en portugais font donc ressortir plusieurs observations. Premièrement, la majorité des enfants (6/10) ne présente pas de taux de production correcte supérieur pour les séquences $\mathrm{Cl}$ comparativement aux séquences $\mathrm{Cr}$. Deuxièmement, l'épenthèse vocalique, bien que peu fréquente, est répertoriée dans une minorité d'exemples (46 sur un total de 498 productions). Finalement, on observe un fort degré de variabilité entre les enfants : un enfant ne produit pratiquement aucune attaque branchante conformément à la cible (PF05); un autre présente des taux élevés pour les deux types d'attaques branchantes (PF03), tout en maintenant des taux de production cible supérieurs pour les séquences $\mathrm{Cl}$ par rapport à $\mathrm{Cr}$, une relation qu'on retrouve aussi chez trois autres enfants (PF01, PF02 et PF04). Enfin, les cinq autres enfants affichent des taux de production quasi similaires, quoique toujours variables, entre ces attaques.

De plus, si on compare les données relatives à la production d'attaques branchantes dans les deux langues évaluées, nous pouvons observer les faits suivants. D'un point de vue global, les enfants produisent plus d'attaques branchantes, conformément à la cible, en français qu'en portugais. Et l'épenthèse vocalique, même si elle est peu attestée, demeure une caractéristique propre au portugais (9\% des cas, comparativement à une seule occurrence en français). Malgré ces généralisations, on observe aussi d'importantes différences entre les enfants, tant qualitatives que quantitatives, incluant celles décrites dans les paragraphes précédents. Dans la prochaine section, nous discutons de ces résultats à la lumière de ceux obtenus dans des études antérieures.

\section{Effets constants et variation entre apprenants}

Dans cette section, nous comparons les données de la présente étude avec celles répertoriées chez des enfants monolingues et bilingues dans 
la littérature antérieure. Dans un premier temps, nous discutons des résultats qui s'appliquent aux dix apprenants bilingues évalués. Dans un second temps, nous traitons de la variation observée entre ces apprenants, en considérant certains facteurs potentiellement responsables de cette variation.

\subsection{Effets constants}

D'un point de vue systématique, nos données indiquent que les attaques branchantes sont acquises plus précocement en français qu'en portugais. Exception faite de PF05, qui n'a acquis cette structure dans aucune de ses deux langues cibles, et de PF03, qui présente des valeurs proches de l'acquisition dans les deux langues, les résultats des huit enfants restants suggèrent une vitesse d'acquisition plus rapide des attaques branchantes en français. Soit les attaques branchantes sont acquises en français et pas en portugais, soit elles sont en cours d'acquisition dans les deux langues, avec des taux de production correcte plus élevés en français qu'en portugais. Ces résultats correspondent à ceux rapportés dans l'étude de cas d'Almeida (2011) ainsi qu'aux différents résultats observés entre les monolingues portugais (Freitas, 1997) et français (Rose, 2000).

De manière similaire, on note une absence quasi-généralisée d'épenthèse dans les données des enfants bilingues, avec la vaste majorité des cas répertoriés en portugais et surtout pour des séquences Cr. Ces données correspondent à la phase de développement des attaques branchantes, c'est-à-dire lorsqu'un enfant arrive à produire des attaques branchantes mais pas de façon systématique. Néanmoins, les épenthèses observées chez les bilingues en portugais sont en apparence moins fréquentes que celles rapportées pour les monolingues portugais. En ce sens, nos données sont similaires à celles de l'enfant bilingue suivie longitudinalement par Almeida (2011).

Enfin, notons que tous les enfants bilingues évalués, lorsqu'ils réduisent l'attaque branchante cible, ont tendance à élider la liquide et à préserver la première consonne. Ces résultats sont similaires à ceux décrits dans la littérature pour les monolingues, peu importe leur langue cible (p. ex., Fikkert 1994; Freitas, 1997; Rose, 2000).

\subsection{Variation des patrons d'acquisition entre apprenants}

En dépit de ces similarités, les enfants étudiés présentent aussi une certaine variation quant à l'influence de la qualité de la liquide sur la 
vitesse d'acquisition de l'attaque branchante. Rappelons que des données longitudinales d'une enfant bilingue suggèrent que les séquences ayant une latérale en deuxième position sont acquises plus précocement que les séquences ayant une rhotique (Almeida, 2011).

Ce facteur n'est évidemment observable que lorsque les attaques branchantes sont en phase d'acquisition : si elles sont acquises, ou si elles ne sont pas du tout produites, il est impossible de déterminer si la qualité de la liquide influence la vitesse de stabilisation des attaques branchantes.

Nos données montrent que, lorsqu'une influence de la qualité de la liquide est observable, elle se manifeste toujours de la même manière : les séquences qui comportent une latérale sont favorisées par rapport à celles comportant une rhotique. Plusieurs enfants présentent ce patron dans notre corpus. Le tableau 7 rend compte des cas pour lesquels les pourcentages de production correcte des séquences $\mathrm{Cl}$ sont plus élevés que ceux des séquences $\mathrm{Cr}$ (un écart d'au moins $25 \%$ ) ainsi que des taux de réussite équivalents pour les deux types de séquences. «---» indique qu'une telle asymétrie n'est pas vérifiable, soit parce que toutes les attaques branchantes sont stables (PF01, PF04, PF06, PF07, PF08, PF09), soit parce qu'aucune attache branchante n'est produite (PF05).

\begin{tabular}{|c|c|c|c|c|c|c|c|c|c|c|}
\hline & PF01 & PF02 & PF03 & PF04 & PF05 & PF06 & PF07 & PF08 & PF09 & PF10 \\
\hline P & $\mathrm{Cl}>\mathrm{Cr}$ & $\mathrm{Cl}>\mathrm{Cr}$ & $\mathrm{Cl}>\mathrm{Cr}$ & $\mathrm{Cl}>\mathrm{Cr}$ & --- & $\mathrm{Cl} \approx \mathrm{Cr}$ & $\mathrm{Cl} \approx \mathrm{Cr}$ & $\mathrm{Cl} \approx \mathrm{Cr}$ & $\mathrm{Cl} \approx \mathrm{Cr}$ & $\mathrm{Cl} \approx \mathrm{Cr}$ \\
\hline $\mathrm{F}$ & --- & $\mathrm{Cl}>\mathrm{Cr}$ & $\mathrm{Cl}>\mathrm{Cr}$ & --- & --- & --- & --- & --- & --- & $\mathrm{Cl}>\mathrm{Cr}$ \\
\hline
\end{tabular}

Tableau 7. - Taux de réussite plus élevé des séquences $\mathrm{Cl}$ par rapport à $\mathrm{Cr}$.

D'une part, en français, trois enfants présentent des taux de productions correctes plus élevés pour $\mathrm{Cl}$ que pour $\mathrm{Cr}$ (PF02, $\mathrm{PF} 03, \mathrm{PF} 10)$. Les données des sept autres enfants ne sont pas exploitables, car soit toutes les attaques branchantes sont déjà acquises, soit elles n'ont pas encore émergé (dans le cas de PF05). D'autre part, en portugais, quatre enfants présentent également ce comportement : PF01, PF02, PF03 et PF04, alors que cinq autres enfants présentent des taux de productions correctes similaires entre les deux langues. Enfin, PF05 ne produit aucune attaque branchante. Ces observations suggèrent que, chez certains enfants, l'acquisition de cette structure est tardive. Cette variation entre les enfants en portugais est aussi comparable à divers résultats répertoriés chez les monolingues : certains enfants développent les deux types d'attaque branchante à la même vitesse (Almeida \& Freitas, 2010) alors 
que d'autres développent plus précocement les séquences $\mathrm{Cl}$ (Mendes, Afonso, Lousada \& Andrade, 2009).

En ce qui concerne l'influence du type de consonne liquide sur le développement des attaques branchantes, seules les données de trois enfants (PF02, PF03 et PF10) sont exploitables en termes de comparaison de ce patron de développement dans les deux langues. Nous observons que deux d'entre eux acquièrent les attaques branchantes avec latérale avant celles contenant une rhotique dans les deux langues (PF02 et PF03), alors que PF10 ne présente ce comportement qu'en français. Les données de PF02 et PF03 suggèrent donc une interaction interlinguistique, conformément aux données de l'enfant bilingue évaluée par Almeida (2011), alors que les résultats de PF10 mènent à poser des patrons de développement autonomes dans chaque langue. Notons, cependant, que seule une observation longitudinale du développement permettrait réellement d'attester de la systématicité des interactions entre les langues de nos enfants bilingues sur une période de temps étendue (Paradis \& Genesee, 1996).

Malgré les limitations inhérentes à notre méthodologie, on observe tout de même que la variation observée quant à l'effet de la liquide ne semble pas être reliée au profil linguistique de nos apprenants. D'une part, des enfants avec profils similaires présentent des comportements variés, alors que, d'autre part, des enfants présentant des comportements similaires peuvent avoir des profils bien distincts. C'est le cas par exemple de PF02 et PF03, qui manifestent une acquisition plus précoce des structures $\mathrm{Cl}$ dans leurs deux langues mais qui présentent des profils distincts : PF02 est dominant en portugais alors que PF03 est considéré comme un bilingue équilibré. Enfin, sur les quatre enfants qui maitrisent les suites $\mathrm{Cl}$ avant les suites $\mathrm{Cr}$ en portugais, on retrouve un dominant français, un dominant portugais et deux bilingues équilibrés. Il ne semble donc pas que le profil de dominance, tel qu'évalué par le questionnaire parental, permette de rendre compte de la variation observée dans l'échantillon étudié. Seule une comparaison avec d'autres groupes de bilingues, soit grandissant en France, soit fréquentant d'autres types d'établissements d'enseignement, par exemple, pourrait nous permettre d'évaluer les impacts potentiels du profil linguistique des enfants bilingues sur leurs patrons de production. Il serait également pertinent d'évaluer des enfants monolingues portugais et français avec les outils utilisés pour notre recueil de données afin de permettre une comparaison plus étroite entre les patrons observés dans des populations bilingues et monolingues. 


\section{Discussion}

Dans cet article, nous avons comparé les données d'enfants bilingues avec celles d'enfants monolingues, lesquelles ont été recueillies à l'aide de différentes méthodes. Nos résultats mettent au jour des patrons constants chez plusieurs enfants bilingues, malgré l'hétérogénéité caractéristique des populations bilingues (De Houwer, 2009). En revanche, la méthode transversale ne permet de rendre entièrement compte ni de parcours développementaux ni de l'ensemble complet des facteurs (grammaticaux ou externes) pouvant influencer le développement phonologique en général, comme par exemple l'influence de la qualité de la liquide sur la production correcte du groupe consonantique, que nous n'avons pu observer avec un détail suffisant que pour deux des dix enfants évalués. De même, les données transversales ne nous ont pas permis de tirer de conclusions sur l'âge de stabilisation des attaques branchantes en portugais. De telles informations sont cependant essentielles pour établir des cas d'interaction linguistique en contexte de développement bilingue. Comme les données transversales ne permettent qu'un cliché momentané des capacités de production des enfants, ce cliché n'est en fait pas interprétable du point de vue du développement de la phonologie. Le chercheur ne peut en fait qu'observer les données recueillies mais ne possède que très peu de moyens de les interpréter d'un point de vue systématique, lequel requiert un accès aux comportements précédents et subséquents à tout patron développemental. Il demeure donc difficile de déterminer si les patrons observés découlent d'influences intrinsèques (p. ex., concernant le développement phonologique général de l'enfant, tant aux niveaux segmental que prosodique), ou extrinsèques (p. ex., relevant de lacunes au niveau articulatoire ou moteur; Inkelas \& Rose, 2007; Rose \& Inkelas, 2011). On peut en fait comparer l'observation d'un individu à l'aide d'un protocole transversal à celle d'un personnage de film dont on ne connait ni le début ni la fin. Ce problème est d'autant plus criant pour les études en contexte de développement bilingue. Une approche transversale comme celle adoptée ici ne permet pas d'évaluer en détail le rythme de développement linguistique de l'enfant dans chacune de ses deux langues, par exemple, ce qui impose plusieurs limites à l'interprétation des données recueillies. Tel est le cas pour sept des dix enfants étudiés ici. De ce point de vue, et considérant aussi le besoin central d'évaluer de larges po-pulations à des fins cliniques, on se doit de préconiser une approche complémentaire pour chacune des méthodes. Le recours à 
une répétition de l'évaluation transversale étalée dans le temps permettrait certainement d'offrir une image plus globale du développement linguistique.

\section{RÉFÉRENCES BIBLIOGRAPHIQUES}

Almeida, Laetitia de. (2011). Acquisition de la structure syllabique en contexte de bilinguisme simultané portugais-français (Thèse de doctorat non publiée). Université de Lisbonne. Disponible en ligne sur <http:// repositorio.ul.pt/handle/10451/4972> (consulté le 25 janvier 2015).

Almeida, Laetitia de. (2013). Un test de phonologie du français standard. Manuscrit. Centre de linguistique de l'Université de Lisbonne.

Almeida, Laetitia de \& Freitas, Maria João. (2010, juillet). Target Phonologies in Bilingual and Monolingual Development of Branching Onsets. Communication présentée au «Workshop Phon \& PhonBank: Future Directions », Memorial University of Newfoundland

Barlow, Jessica A. (1997). A Constraint-Based Account of Syllable Onsets: Evidence from Developing Systems (Thèse de doctorat). Indiana University.

Bérubé, Daniel, Bernhardt, B. May \& Stemberger, Joseph P. (2013). Un test de phonologie du français : construction et utilisation. Canadian Journal of Speech-Language Pathology and Audiology / Revue canadienne d'orthophonie et d'audiologie, 37(1), 26-40.

Bérubé, Daniel, Bernhardt, B. May, Stemberger, Joseph P. \& Almeida, Laetitia de. (2014). Un test de phonologie du français (canadien et standard) : construction et utilisation. Orthophonies, 16, 14-56.

De Houwer, Annick. (2009). Bilingual First Language Acquisition. Clevedon/Buffalo : Multilingual Matters Limited.

Fabiano-Smith, Leah \& BARLow, Jessica A. (2009). Interaction in Bilingual Phonological Acquisition: Evidence from Phonetic Inventories. International Journal of Bilingual Education and Bilingualism, 13(1), $1-17$.

FiKKeRt, Paula (1994). On the Acquisition of Prosodic Structure. The Hague : Holland Academic Graphics.

Freitas, Maria João. (1997). Aquisição da Estrutura Silábica do Português (Thèse de doctorat). Université de Lisbonne.

Freitas, Maria João. (2003). The Acquisition of Onset Clusters in European Portuguese. Probus, 15, 27-46.

GoAd, Heather \& Rose, Yvan. (2004). Input Elaboration, Head Faithfulness and Evidence for Representation in the Acquisition of Left-edge Clusters in West Germanic. Dans R. Kager, J. Pater \& W. Zonneveld (dir.), 
Constraints in Phonological Acquisition (p. 109-157). Cambridge : Cambridge University Press.

Grosjean, François. (2004). Studying Bilinguals: Methodological and Conceptual Issues. Dans Tej K. Bhatia \& William C. Ritchie (dir.), The Handbook of Bilingualism (p. 32-64). Oxford : Blackwell.

Inkelas, Sharon \& Rose, Yvan. (2007). Positional Neutralization: A Case Study from Child Language. Language, 83(4), 707-736.

Kehoe, Margaret, Hilaire-Debove, Géraldine, Demuth, Katherine \& Lleó, Conxita. (2008). The Structure of Branching Onsets and Rising Diphtongs: Evidence from the Acquisition of French and Spanish. Language Acquisition, 15, 5-57.

Lleó, Conxita. (2002). The Role of Markedness in the Acquisition of Complex Prosodic Structures by German-Spanish Bilinguals. International Journal of Bilingualism, 6, 291-313.

Mateus, Maria H. \& D'Andrade, Ernesto. (2000). The Phonology of Portuguese. Oxford : Oxford University Press.

Mendes, Ana, Afonso, Elisabete, Lousada, Marisa \& Andrade, Fátima. (2009). Alpe-Avaliação da Linguagem Pré-escolar, Teste FonéticoFonológico. Aveiro : Designeed.

Müller, Natascha \& Hulk, Aafke. (2001). Crosslinguistic Influence in Bilingual Language Acquisition: Italian and French as Recipient Languages. Bilingualism: Language and Cognition, 4(1), 1-21.

Paradis, Johanne \& Genesee, Fred. (1996). Syntactic Acquisition in Bilingual Children: Autonomous or Interdependent? Studies in Second Language Acquisition, 18, 1-25.

Ramalho, Margarida, Almeida, Laetitia de \& Freitas, Maria João. (2013). CLCP - PE (Avaliação Fonológica da Criança: Crosslinguistic Child Phonology Project - Português Europeu). IGAC 4469.

Rice, Keren. (1992). On Deriving Sonority: A Structural Account of Sonority Relationships. Phonology, 9, 61-99.

Rose, Yvan. (2000). Headedness and Prosodic Licensing in the L1 Acquisition of Phonology (Thèse de doctorat). McGill University.

Rose, Yvan \& Inkelas, Sharon. (2011). The Interpretation of Phonological Patterns in First Language Acquisition. Dans C. J. Ewen, E. Hume, M. van Oostendorp \& K. Rice (dir.), The Blackwell Companion to Phonology (p. 2414-2438). Malden, MA : Wiley-Blackwell.

Rose, Yvan \& MacWhinney, B. (2014). The PhonBank Initiative. Dans J. Durand, U. Gut \& G. Kristoffersen (dir.), The Oxford Handbook of Corpus Phonology (p. 380-401). Oxford : Oxford University Press.

Rose, Yvan, MacWhinney, Brian, Byrne, Rodrigue, Hedlund, Gregory, Maddocks, Keith, O’Brien, Philip \& Wareham, Todd (2006). Introducing Phon: A Software Solution for the Study of Phonological Acqui- 
sition. Dans D. Bamman, T. Magnitskaia \& C. Zaller (dir.), Proceedings of the 30th Annual Boston University Conference on Language Development (p. 489-500). Somerville, MA : Cascadilla Press.

Santos, Christophe dos. (2007). Développement phonologique en français langue maternelle : une étude de cas (Thèse de doctorat). Université Lumière Lyon 2.

Smith, Neil V. (1973). The Acquisition of Phonology: A Case Study. Cambridge : Cambridge University Press.

Spencer, Andrew. (1986). Towards a Theory of Phonological Development. Lingua, 68, 3-38.

\section{ANNEXE}

1. Liste des mots cibles contenant des attaques branchantes de l'outil français (Almeida, 2013) :

\begin{tabular}{|c|c|c|c|}
\hline $\begin{array}{l}\text { Groupe } \\
\text { /pr/ }\end{array}$ & $\begin{array}{l}\text { Mot } \\
\text { princesse }\end{array}$ & $\begin{array}{l}\text { Groupe } \\
\text { /pl/ }\end{array}$ & $\begin{array}{l}\text { Mot } \\
\text { plume } \\
\text { plage } \\
\text { parapluie } \\
\text { plonger }\end{array}$ \\
\hline /br/ & $\begin{array}{l}\text { bras } \\
\text { bruit }\end{array}$ & $/ \mathrm{bl} /$ & $\begin{array}{l}\text { bleu } \\
\text { blanc }\end{array}$ \\
\hline$/ \mathrm{tr} /$ & $\begin{array}{l}\text { triste } \\
\text { trois } \\
\text { train } \\
\text { tracteur } \\
\text { citrouille }\end{array}$ & & \\
\hline$/ \mathrm{dr} /$ & $\begin{array}{l}\text { drapeau } \\
\text { dragon }\end{array}$ & & \\
\hline$/ \mathrm{kr} /$ & $\begin{array}{l}\text { crayon } \\
\text { crocodile }\end{array}$ & $/ \mathrm{kl} /$ & $\begin{array}{l}\text { clown } \\
\text { cloche }\end{array}$ \\
\hline /gr/ & $\begin{array}{l}\text { grand } \\
\text { grenouille }\end{array}$ & $/ \mathrm{gl} /$ & glace \\
\hline /fr/ & $\begin{array}{l}\text { fraise } \\
\text { froid } \\
\text { fruit } \\
\text { dentifrice }\end{array}$ & $/ \mathrm{fl} /$ & $\begin{array}{l}\text { fleur } \\
\text { flûte }\end{array}$ \\
\hline
\end{tabular}

2. Liste des mots cibles contenant des attaques branchantes de l'outil portugais (Ramalho, Almeida \& Freitas, 2013) : 


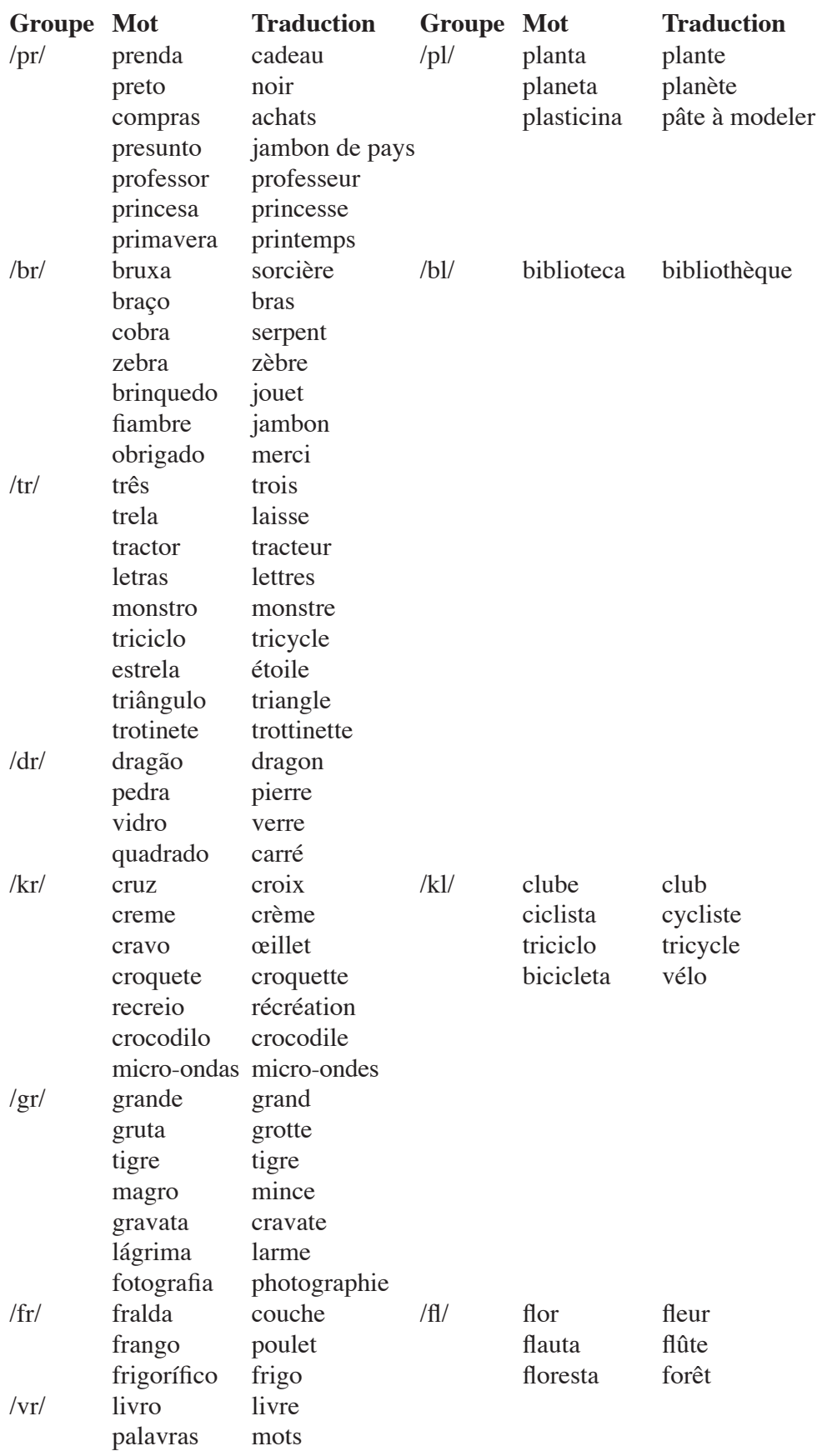

